\title{
Foregut Carcinoid Tumor
}

National Cancer Institute

\section{Source}

National Cancer Institute. Foregut Carcinoid Tumor. NCI Thesaurus. Code C6421.

A carcinoid tumor that arises from the lung, thymus, esophagus, stomach, or duodenum. 